\title{
Green Cloud Computing: A Constructive Advent To Curtail The Carbon Emission Radiation Due To Servers.
}

\author{
PalakMakhija $^{* 1}$, ShrutiAgarwal ${ }^{* 2}$, Monali Jindal ${ }^{* 3}$, Niyati Gupta ${ }^{* 4}$ \\ ${ }^{*}$ Computer Science and Engineering Department, Suresh GyanViharUniversity,MahalJagatpura, Jaipur, India.
}

\begin{abstract}
This paper analytically scrutinizes the energy dissipation based on services and invades the conditions to promote green cloud computing to save energy crisis. In conventional, cloud computing emphasis on the data computing efficiency whereas green cloud computing accredit to the research and practice of applying computing resources in virtuous, adequate and commercial way.

At present, the cloud computing systems waste an enormous volume of energy and large amount of carbon dioxide are substantially emitted. The extensive ejections of carbons are primarily due to heat generated by the data centre servers.Information systems that are organized are needed to be amended directly. Through admittance to remote servers accumulated in "green data centres" disbursed by the service providers, employees in the company are endowed with lighter pcs. The pivot able aspect of green cloud computing is to asset the approach of decussating the desolation of power resources. The intent of green computing is analogous to green chemistry; aggrandize the energy adequacy in the midst of product's lifetime, pauperize the adoption of precarious component.
\end{abstract}

Keywords: Green Virtual network, energy aware routing, global Green Cloud Computing, warming.

\section{Introduction}

The alarming upsurge in the convention of thousands of concomitant e-commerce transactions and lots of web queries a day is due to the beefing up of large speed networks. The ever proliferating demand is handled via large scale datacentres, which amalgamate many numbers of servers with other infrastructures like network system, cooling and storage[1]. These datacentres are operated by many internet companies such as Wikipedia, Yahoo and Google around the world.Cloud computing is defined as the commercialization of these advancement, where computing is conveyed as adequacy on a pay- as- you -go basis. Traditionally, a stupendous amount of money and time are infused by business organizations in procurement and subsistence of computational resources. The ownership based approach is swiftly diminishing into subscription oriented approach due to the emanation of Cloud Computing by bestowing access to services on demand and spreading foundation. Any amount ofinformation can be accumulated and shared by the users [2].

Diminutive organizations need not to woe about the buying, administering and configuring their own computing foundations. They can concentrate on cuspidation of their gist expertise by exploiting a number of godsends of Cloud Computing like expeditious and moderate software development at low charges. Furthermore, Cloud Computing also propose tremendous amount of power to process excessive amount of data produced every day. Nevertheless, the increasing demand of Cloud infrastructure has surpassingly augmented the energy devastation of data centres, which has become a censorious matter of contention. The main hindrance in the progress of Cloud Computing is that there is large amount of carbon emission which is not felicitous with the environment and in addition render to high translation cost which abate the advancement in the profit of cloud providers[3]. Hence, in order to curtail the impact of Cloud Computing over the environment some energy efficient solutions need to be implemented.

Resource virtualization and work consolidation are some of the technologies that can be make data centres more efficient to energy but this will not work efficiently for the next few years as some studies on Cloud Computing detect that exasperate the problem of global warming and carbon emission as the computing resources are collectively demanded. Even the most conveniently build data centre with the maximum utilization rate can extenuate, instead eliminate, co2 emissions [4]. The reason behind is that the main aim of cloud provider is to reduce the electricity cost rather than carbon emission. As, cloud computing is an emerging technology also put up considerable questions like whether it is sustainable to environment or not. Thus, this chapter scrutinizes the various solutions related to $\mathrm{co} 2$ emission problem by considering various emerging technologies which is crucial for users and organizations implementing Cloud Computing.

Finally, we also affirm and eulogize a Green Cloud Computing framework for decreasing its carbon footprint in the invigorated manner without enduring the quality of service (availability and performance). 


\section{Green Computing}

Green computing is the study and usage of proficient and eco-friendly computing resources which is now beneath the diligence of not only the businesses of other industries but also the environmental organizations. Going green is the best interest of the companies in the computer industry that they have realized in the recent years, as in the terms of reduced costs and public interest[5].

The stream of "Green Technology" encloses wide range of subjects from the study of advanced equipments that are used in our daily routine to the latest energy formation techniques. Green technology target on depreciating the environmental percussion of the industrial process and leading-edge technologies generated by the earth's increasing population. The goal provided to the society's needs that does not damage or devour natural resources is taken by green technology. This means to create the recyclable products fully, decreasing pollution, adducing flipside technologies in numerous fields, and concocting the centre of commercial actions around the technologies that avail the environment.

The enormous amount of computing fabricated worldwide has a absolute percussion on environment issues, and numerous studies conducted by the scientist is to reduce the reluctant impact on our natural resources.

\section{Green It}

The heart of every blooming modern business is the Information Technology (IT). The enforcement of Green IT through the energy efficiency has migrated to centre phase for a lot of companies in their quest of helping make a idiosyncrasy for the environment.

Making momentous steps in the green order for numerous reasons for companies Green IT is the ideal way for it. First, to remain the competitive order of the company IT should be refreshed continually as a sense of company policy. Substituting the IT equipment and elevating diligence architecture with energy efficacious system like virtual data storage, virtual servers and database structure and efficient application can simply recede IT power utilization for the redressed equipment's. The second reason responsible for green IT is that virtual data storage technology and virtual servers are modus that allow reducing system management and equipment costs for data centres.

The "green" concept for creating Information Technology green is powerful that helps in reducing greenhouse gases and thus reduce global warming.

\section{Cloud Computing}

\section{Virtualization- A Boon For}

Virtualization is one of the important approaches of Cloud Computing. Virtualization accomplishes cogitation of resources, both in software and hardware, in order to imitate multiple multifarious execution environments in computer system. Virtualization also help to curtail the numbers of equipments required in IT hardware i.e. it makes an adequate use of assets and energy. In virtualization, the single hardware exercise as multiple parts [6].

Different end users are confined to different hardware parts hence each part of hardware work as distinctive entity. Establishing virtual function infrastructure in cloud, fewer number of servers are used to run applications and different operating systems, which results in the devastation of overall energy utilised by the data centres and hence helps in their cooling. The reduction in the number of server results in attrition of data centre building size. Illustrating some of the eminence of virtualization which directly impacts the environment is as follows:

1. Migration of virtual machine from one server to another can eradicate the planned downtime.

2. It procures automated failover for applications that are virtualised and vigorously balance workload on server.

3. Managed and supervised resource allotment.

4. Server's group ability to share resources is exponentially increased by virtualization.

In Cloud Computing, the resource provision can be elucidated by network abstraction which is offered by network virtualization. Beyond cognizance for the underlying network infrastructure, the Cloud Computing operator can extend the services over the virtual network. A customized virtual network can be owned by each service. Hence, all the components of QoS are fulfilled. Different networks can run concurrently in a same physical infrastructure without meddling with each other.

Storage virtualization makes use of software and hardware to rift the association storage subsystem and system service, application component and application. This acquiesce the depository to be located anywhere, on any device duplicated for performance and reliable reasons. 


\section{Energy Aware Routing}

The objective of the data centre network is to affix the large number of data centre servers, thus provide efficacious and fault-tolerant applicability to upper layer applications. To save the consumption of energy in high density data centres "energy aware routing "is used. The basic concept is to use less network devices to dispense the routing services as hopeful, with small cede on the network performance. To save energy we have to shut down the idle network device or put into sleep mode[7].

A new data centre architectures common characteristic is that they disclose much richer link connectivity. However in busy-hour- load or worst-case like when all are communicating the high network capacity supremelyprovisioned. The traffic of the data centre is below the peak value at most of the time. Especially between the daytime and night time the traffic of the data centre varies. During the day time the traffic is at peak and falls at the night. So, the large number of network devices in highly connected data centre networks works idles.

At the similar time, the power-hungry devices that consumed energy become a problem for many data centres owner. Ideally when there is ideal switch than no power consumption and when network load increases energy consumption also increases. Unluckily, the network devices that we use today are not energy propositional [8].

\section{Solution to the Problem of Data Centre in Cloud Computing}

The enormous ecological degradation provocation today is global warming, which is due to the emission of carbon. Green Computing is brought by energy predicament, which furthers needs some methods and principle to be generated for energy efficiency. The distinctive approaches are recycling, resource allocation, environment friendly web hosting, reduce, grid computing[9]. They are explained below:

1. Recycling: Recycling is defined as the process of making use of unwanted products in order to manufacture the new products. The components that are left after the new products are remanufactured are acknowledged as crucial. There are few entities which cannot be remanufactured easily like mobile phones, old personal computers, which makes reuse of elements quite difficult and challengeable. Other products like tin, plastic, paper are comparatively easy to reuse and hence make the process of recycling process more efficient.The most general reason for the process of recycling to be essential is that the natural resources which are used by new manufactured products are depreciated.

2. Environment friendly web hosting: The best agenda to implement eco-friendly web hosting is to use solar wind in order to keep the operation running, also known as solar power web hosting. We should use the products which are good to environment and does not degrade it, termed as "eco-friendly products". These products contribute to green living which helps in preserving natural resources like air, water and energy.

3. Grid Computing: Grid Computing is defined as the amalgamation of computer resources from multifarious authority to grasp same objective. It is basically used to solve technical and scientific problem which desire high processing speed.system and a massive amount of data. The best example of grid computing is SETI (Search for Extra-terrestrial Intelligence). Grid Computing aspire to advertise betterment and evolution of technologies by which broad area distribute resources achieve scalable and seamless access.

\section{Conclusion}

Task association especially in clouds has evolve into the most important Avenue to assemble resource usage which in turn improves efficiency of energy. As we know that utilization of resource directly recite to energy dissipation, so the result of the study green cloud computing should not only have absolute strike on the electricity bills minimization of cloud foundation providers, but also entail possible savings in different operations.

So from now onwards data centre are to be placed in appropriate place so that it provides a solution for the reduction of heat. The usage ofCPU are at the maximum level and the enhancement of the system requirement is based on the goal need to perform and the rebuttal is based on cloud computing and grid computing. By the cloud computing report of carbon consumption and carbon emission it is realized that future is totally based on cloud computing.

Enduring the quest of world fiscal development and opulence, incommensurate load in the atmosphere, counterattacks abide to aggrandize, a gigantic attention has been thrown over the problem of climate change and global warming to the various artiste and research scholars. It has become extremely crucial to concentrate on the environment friendly management of economic activities. Further, the process required for the business enhancement has its own righteous boundation to dodge excessive scarcity of essential resources, the impact of which results in enormous difficulties, the expertise must take initiative on their own and think inventive methodology to minimize carbon emission. In order to make a step towards the substantial living and green atmosphere, government should endorse the approaches to curtail the carbon emission. 


\section{References}

[1]. Gartener, "Data Centres Consolidation": Trends and Best Practices, http, 2010.

[2]. S.Srikantaiah, A.Kansal, and F.Zhao, "Energy aware consolidation for cloud computing," Proc the 2008 conference on Power aware computing and system (Hot Power'08), CA, USA, 2008.

[3]. "Gartener Estimates ICT Industry Accounts for 2 Percent of global CO2 Emissionshttp://www.gartner.com/it/page.jdp?id=503867, Available the March 2011.

[4]. Char-Dir Chung, Ten-Chu kao, Jia-Xu Yang, Cloud Computing and Industrial Development, Research, Development, and Evaluation Commission, Executive Yuan, Taiwan, Research and Evaluation, Bimonthly, 34(4). August 2010. 20-31. (in Chinese).

[5]. The green grid consortium (2011)

[6]. S. Bhatia, M. Motiwala, W. Muhlbauer, Y. Mundada, V. Valancius, A.Bavier, N. Feamster, L. Peterson, and 1. Rexford, "Trellis: A platform for building flexible, fast virtual networks on commodity hardware," Proceedings of Workshop on Real Overlays and Distributed Systems.

[7]. http://conferences.sigcomm.org/sigcomm/2010/papers/green/p1.pdf

[8]. U.S. Environmental Protection Agency. DataCenter Report to Congress www.energystar.gov

[9]. Chih-Chien Liu, Industry of Green products and green management strategy - the Directive Method of EU RoHS, WEEE, Taiwan Watch ,6(4). 2004. 45-58. (In Chinese) 